\title{
A Decision Problem of Hotels on Setting Online-Exclusive-Rooms in E-Commerce Age
}

\author{
Liang Xu, Xiaoyan Xu \\ University of Science and Technology of China, Hefei, China \\ Email: xliang16@mail.ustc.edu.cn, xxy204@ustc.edu.cn
}

Received 6 June 2014; revised 2 July 2014; accepted 28 July 2014

Copyright (C) 2014 by authors and Scientific Research Publishing Inc.

This work is licensed under the Creative Commons Attribution International License (CC BY). http://creativecommons.org/licenses/by/4.0/

(c) (i) Open Access

\begin{abstract}
Due to the rapid development of information technology, customers' consumption pattern in hotel industry has dramatically changed, and many people book rooms through some professional online platforms. Hotel managers need to redesign their sale channel structures by incorporating online platforms, in order to increase profit. This paper focuses on a revenue maximization problem for a hotel which cooperates with an online platform. Two cooperating forms are investigated and compared. Specifically, one intuitive form is named complete sharing, in which all the hotel rooms are shared with the online platform for booking. The other form is named setting online-exclusive-rooms (OERs), in which the hotel set a fixed quantity of rooms for exclusively booking from the online platform. By building a loss queueing model, this paper finds some properties of the optimal quantity of OERs in the latter form. Factors affecting the selection and how they affect the selection are also investigated.
\end{abstract}

\section{Keywords}

Revenue Management, Hotel Room Management, Online Platform, Optimization

\section{Introduction}

During the last two decades, information technology has been developing rapidly. More and more people buy products or book service through the internet [1]. A typical example is that many customers prefer to booking hotel rooms via online platforms (e.g. Expedia, CTRIP, etc.), rather than through the front desk of the hotel. Customers' consumption pattern has changed and is still changing hotel industry. According to CTRIP's survey in 2010, more than $40 \%$ of hotel customers book rooms through such online platform in China, and more than 1 million rooms are booked through CTRIP per year [2]. Professional hotel booking platforms nowadays have become an important channel for hotel room booking [3] [4]. 
With this emerging phenomenon, hotel managers have to redesign their sale channel structures by incorporating online platforms, in order to reach different market segments, expand market share, and increase profit. One intuitive cooperating form between a hotel and an online platform is that, the hotel completely shares all rooms with the online platform for room booking. The online platform gets a commission from the hotel once it successfully accomplishes a room booking. We name this cooperating form as "complete sharing". This form seems to be reasonable and can maximize the utilization of the hotel rooms. However, this cooperating form may lead to a foreseeable result for the hotel: more and more customers will choose to book rooms via the online platform, and then the hotel should strongly rely on the online platform for getting customers. In the long term, the profit of the hotel cannot be maximized. Actually, the cooperation between CTRIP and many hotels is in another form. The hotel offers a fixed quantity of rooms to the online platform (i.e. CTRIP) which is less than the total quantity of rooms the hotel has. This fixed quantity of rooms can only be booked from the online platform, so that we call them Online-Exclusive-Rooms (OERs). No more room can be booked from the online platform once all OERs have been booked out. We name this cooperating form as "setting OERs".

In this paper, we focus on a profit maximization decision problem for a hotel when cooperating with an online platform. Specifically, we intend to answer the question that should a hotel set OERs, and if yes, how many OERs should be set. The rest of this paper is structured as follows. In Section 2, we review the related literature and show the difference between our work and the extant research, where the contributions of this research are addressed. In Section 3, the model is formally introduced, and some properties of the optimal quantity of OERs are discussed. In Section 4, we formally discuss how parameters affect the optimal quantity of OERs and the selection of cooperating forms, by analytical study and numerical study. We conclude this paper and give some future research directions in Section 5. All the proofs of propositions are relegated to the Appendix.

\section{Literature Review}

Our work is relevant to the research on hotel yield management and the dual-channel supply chain management. However, there are some significant differences between this work and the extant literature. Next we will review the literature and the differences accordingly.

\subsection{Hotel Yield Management}

Much of the early literature in hotel yield management focuses on the overbooking problem, i.e. to set appropriate overbooking limits, e.g., see Rothstein [5], Liberman and Yechialli [6]. Later on, the literature on yield management focuses on maximizing the profit generated by a given capacity for service in the long run [7] [8]. In hotel industry, the capacity is the number of rooms the hotel has and the operational objective in hotel yield management is to sell the right rooms to the right customers at the right price. Bitran and Mondschein study optimal strategies for renting hotel rooms when there is a stochastic and dynamic arrival of customers from different market segments [9]. They formulate the problem as a stochastic and dynamic programming model and characterize the optimal policies as functions of the capacity and the time left until the end of the planning horizon. Bitran and Gilbert formulate a room allocation problem as a stochastic dynamic program and develop simple heuristic procedures for accepting reservations [10]. Badinelli also presents a dynamic programming formulation of the hotel yield management problem allowing for general demand patterns and a policy that is based on time and the number of vacancies [8]. Baker and Collier investigate a new Price Setting Method (PSM) to manage demand in the hotel yield management [11].

The main difference between the classical hotel yield management research and our work is that, the capacity (rooms) is usually regarded as a given resource constraint in classical hotel yield management research but a decision variable in our problem. Accompanied by the coming of E-commerce age, the problem of designing an optimal cooperating mechanism for hotel room booking service has emerged, but not investigated yet in the literature. In addition, an operational difficulty in hotel yield management is the customer's multiple night stays [9]. Generally, this problem is approximated by a series of independent single night reservations in extant hotel yield management literatures [10]. In our work, this difficulty does not exist, because the customers' stay time can be any number of days.

\subsection{Dual-Channel Supply Chain Management}

Most of the traditional dual-channel supply chain management research focuses on selling tangible product such 
as computer, which can be stored for selling at different times. The rapid development of E-commerce has made it easier for tangible product manufacturers to move part of sales online. About ten years ago, about $42 \%$ of top-ranked suppliers in a variety of industries (e.g., IBM, Pioneer Electronics, Cisco System, Estee Lauder, and Nike) have begun to sell products directly to consumers over the Internet [12]. Manufacturers nowadays are increasingly adopting a dual-channel way to sell their products, i.e., a traditional retail channel and an online channel.

Many problems regarding Dual-channel supply chain management have been extensively investigated in the literature, including the pricing and ordering decision problems in each channel, the coordination and competition problems between the dual channels [13]-[15]. Different from the extant research on traditional dual-channel supply chain management, this work considers the allocation problem of sellable "product" (the room booking service) and the corresponding profit maximizing problem.

In summary, up to now, little is known about how the hotel should use their limited room resource effectively to cooperate with the online platform. This is the main motivation for our work. The main contributions of our paper are three-folds. 1) We study the hotel room allocation problem considering the cooperation between hotel and the online platform. 2) We creatively formulate the loss-queueing model for the hotel service system which consists of a hotel and an online platform. 3) We investigate whether a hotel should set OERs and the property of the optimal quantity of OERs to maximize the hotel's revenue, which provides a theoretical guide for the management of hotel rooms in E-commerce age.

\section{Model Formulation and Analysis}

\subsection{Problem Description}

Consider a hotel service system which is composed of a hotel and an online platform. The hotel provides room service for customers, while any customer can book the room service from either the front desk or the online platform.

Here we only describe the decision problem of the hotel in "setting OERs" case. Take CTRIP as an example. As an online platform, CTRIP provides room booking service to customers for many hotels. Each hotel sets a certain quantity of rooms for booking exclusively from CTRIP, and the remaining quantity of rooms for booking exclusively from the front desk. As addressed in the first section, the former type of rooms are named "Online-Exclusive-Rooms" or "OERs", and the latter "hotel exclusive rooms" or "HERs”. Once CTRIP successfully accomplishes a booking task to one customer, the hotel pays a commission to CTRIP. If CTRIP did not "book out" all its OERs, it does not have any penalty. If CTRIP has "booked out" all its OERs, no extra customer can book a room from CTRIP. Also, if the hotel has "booked out" all its HERs, it cannot accept more customers from the front desk. From this way, it will be preferable for CTRIP to have more OERs, because more OERs bring more opportunities to make money. However, the hotel needs to think about an appropriate quantity of OERs. If the quantity of OERs is too large, the quantity of HERs will be less, which leads to potential loss of the hotel's profit. If the quantity of OERs is too few, it will also lead to potential loss of customers and profit from online platform.

In this paper, we focus on the decision problem of a hotel which cooperates with an online platform. Specifically, we intend to answer the following two questions. First, within the above mentioned two cooperating forms (complete sharing and setting OERs), which one is better and when? In other words, when should a hotel set OERs? Second, in the latter form, how many OERs should the hotel set to maximize its profit? This is actually an optimization problem of the hotel. Since the second question is only related to the second cooperating form (setting OERs), we set the "complete sharing" case as benchmark, and mainly discuss the "setting OERs" case.

For simplicity and convenience of discussion, we make the following assumptions in this work.

1) All the hotel rooms are homogeneous, or of a single type. Actually, hotels usually have multiple types of rooms. For these hotels, different type of rooms can be analyzed separately.

2) Each customer only books one room at one time. This assumption is feasible, since it is the number of rooms booked that affects the revenue of the hotel, not the number of customers who make the booking.

3) There is no refund for the booking. Making this assumption is for simplicity.

4) The online platform does not set the lower threshold of OER quantity, since it will make money even if there is only one OER. In actual case, there may be a lower threshold set by the online platform. However, this constraint can be easily added to the hotel's optimal OER quantity to give the final solution. 


\subsection{Model for "Setting OERs"}

The hotel service system investigated in this paper can be modeled by a queuing service system with multiple servers. The rooms (including OERs and HERs) can be regarded as the servers, and the whole process that customers stay in the hotel rooms can be regarded as the serve process. In our problem, there are two customer flows, one arriving at the hotel's front desk and the other arriving at the online platform. We assume that these two customer flows are independent, and each of them arrives according to a Poisson processes [5] [9] [10]. Assume that the time (days) a customer stay in the hotel (either in an OER or an HER) is an independent and identically distributed (i.i.d.) random variable obeying a general distribution, and independent of the arrival process [16] [17]. Here are the notations used in this paper.

$Q$ : the total quantity of rooms in the hotel;

$q$ : the total quantity of OERs;

$w_{e}$ : the price when a customer books a room from the online platform;

$w_{h}$ : the price when a customer books a room from the front desk;

$r$ : the commission the hotel pays to the online platform for successfully booking out a OER; we suppose that the commission is a proportion of the booking price from the online platform;

$\theta$ : the proportion of the booking price as the commission $(0<\theta<1)$; thus $r=\theta \cdot w_{e}$;

$\lambda_{h}$ : customers' arriving rate at the front desk (obey a Poisson process); independent of the booking prices;

$\lambda_{e}$ : customers' arriving rate at the online platform (obey a Poisson process); independent of the booking prices;

$u$ : service rate in the hotel (obey a general distribution); thus $1 / \mu$ is the expected customer stay time (days) in the hotel.

Following the assumptions and notations above, there are $Q-q$ rooms (HERs) that can be booked from the front desk, and $q$ rooms (OERs) from the online platform. We also note that new arriving customers will leave immediately when all the rooms (either OERs or HERs) are occupied (i.e., all servers are busy). Therefore, the hotel front desk can be regarded as an $M / G / Q-q / Q-q$ queuing system [18], and the online platform can be regarded as an $M / G / q / q$ queuing system. These two systems are independent. We illustrate them in Figure 1.

To answer the two questions raised in Section 3.1, first we need to compute the revenue of the hotel under any quantity of OERs. According to the classical queueing theory [19], the revenue of the service system depends on the expected number of customers in the service system, which in turn depends on the probabilities of all rooms taken in our problem.

Let $P_{h}(q)$ and $P_{e}(q)$ be the probabilities of all rooms taken at front desk and at online platform, respectively, when the quantity of OERs is $q$. Actually, $P_{h}(q)$ and $P_{e}(q)$ also represent the customer loss probability in the $M / G / Q-q / Q-q$ and $M / G / q / q$ systems, respectively. Thus $P_{h}(q)$ and $P_{e}(q)$ can be easily computed as follows [20].

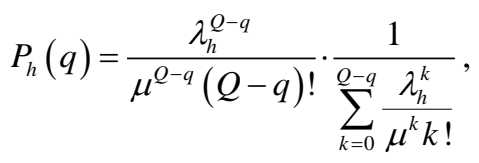

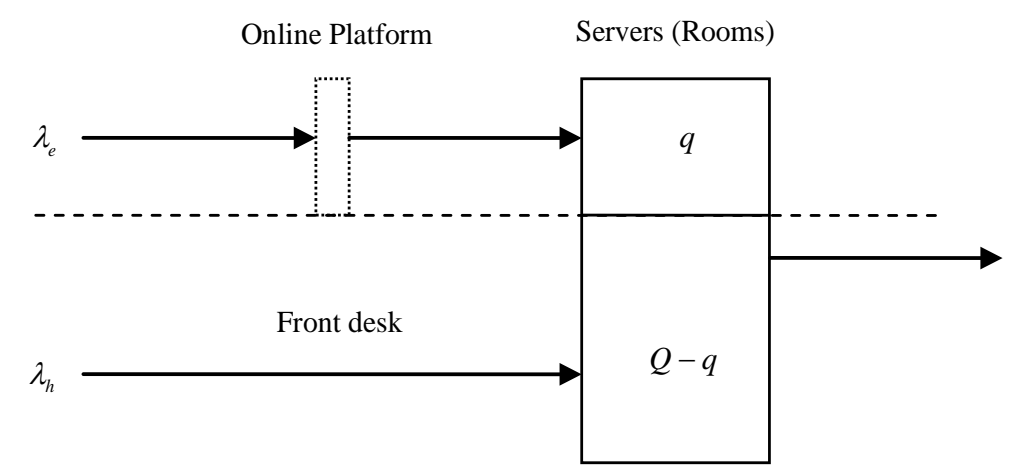

Figure 1. The front desk and online platform queuing service systems. 


$$
P_{e}(q)=\frac{\lambda_{e}^{q}}{\mu^{q} q !} \cdot \frac{1}{\sum_{k=0}^{q} \frac{\lambda_{e}^{k}}{\mu^{k} k !}} .
$$

Let $L_{h}(q)$ and $L_{e}(q)$ be the expected number of customers in the $M / G / Q-q / Q-q$ and $M / G / q / q$ system, respectively, when the quantity of OERs is $q$. Then $L_{h}(q)$ and $L_{e}(q)$ can be calculated as follows [21].

$$
\begin{aligned}
& L_{h}(q)=\frac{\lambda_{h}}{\mu}\left[1-P_{h}(q)\right], \\
& L_{e}(q)=\frac{\lambda_{e}}{\mu}\left[1-P_{e}(q)\right] .
\end{aligned}
$$

The revenue of the hotel comes from two parts: one from the $M / G / Q-q / Q-q$ system and the other from $M / G / q / q$ system (removing the commission paid to the online platform). The expected revenue of the hotel, $\pi_{h}(q)$, is as follows.

$$
\pi_{h}(q)=w_{h} L_{h}+(1-\theta) w_{e} L_{e}=\frac{w_{h} \lambda_{h}}{\mu}\left[1-P_{h}(q)\right]+\frac{(1-\theta) w_{e} \lambda_{e}}{\mu}\left[1-P_{e}(q)\right] .
$$

By substituting (1) and (2) into the Equation (5), we get

$$
\pi_{h}(q)=\frac{w_{h} \lambda_{h}}{\mu}\left[1-\frac{\lambda_{h}^{Q-q}}{\mu^{Q-q}(Q-q) !} \frac{1}{\sum_{k=0}^{Q-q} \frac{\lambda_{h}^{k}}{\mu^{k} k !}}\right]+\frac{(1-\theta) w_{e} \lambda_{e}}{\mu}\left(1-\frac{\lambda_{e}^{q}}{\mu^{q} q !} \frac{1}{\sum_{k=0}^{q} \frac{\lambda_{e}^{k}}{\mu^{k} k !}}\right) .
$$

Since the cost of providing room service can be regarded as a constant, it does not affect the optimal decision of the hotel. In other words, revenue maximization has the same meaning of profit maximization. In the latter part of this paper, we use revenue maximization as the operational objective of the hotel.

Before searching for the optimal decision, we need to understand the property of the expected revenue of the hotel. We proved that the expected revenue of the hotel has the following property, as shown in Proposition 1. All the proofs of propositions are arranged in the Appendix part.

Proposition 1. The expected revenue of the hotel, $\pi_{h}(q)$, is a concave function of the quantity of OERs, $q$.

Proposition 1 implies that, over any interval of the quantity of OERs $(q)$, there is a maximal value of the expected revenue of the hotel (i.e. $\pi_{h}(q)$ ). The optimal quantity of OERs corresponding to the maximal value of $\pi_{h}(q)$ is what we are searching for.

\subsection{The Optimal Quantity of OERs}

Based on Proposition 1, we can formulate the following model to find the optimal quantity of OERs to maximize the hotel's revenue.

$$
\max \pi_{h}(q) \text { s.t. } 0 \leq q \leq Q, \quad q \in \boldsymbol{Z}
$$

Because $q$ is discrete, it is not easy to directly solve the above model. Denote the solution of Model (7) (i.e. the optimal quantity of OERs) to be $q^{*}$. According to Proposition $1, \pi_{h}(q)$ is a concave function of $q$, therefore we can use the marginal analysis method to find $q^{*}$ [22]. In Model (7), $q^{*}$ must satisfy the following conditions:

$$
\left\{\begin{array}{l}
\pi_{h}\left(q^{*}\right) \geq \pi_{h}\left(q^{*}-1\right) \\
\pi_{h}\left(q^{*}\right) \geq \pi_{h}\left(q^{*}+1\right),
\end{array}\right.
$$

where $\pi_{h}\left(q^{*}\right)$ is the expected revenue of the hotel when the quantity of OERs is $q^{*}$. Based on the conditions 
in (8), we have Proposition 2 for determining $q^{*}$.

Proposition 2. The optimal quantity of OERs $\left(q^{*}\right)$ can be determined by the following conditions

$$
\frac{P_{h}\left(q^{*}\right)-P_{h}\left(q^{*}-1\right)}{P_{e}\left(q^{*}-1\right)-P_{e}\left(q^{*}\right)} \leq \frac{(1-\theta) w_{e} \lambda_{e}}{w_{h} \lambda_{h}} \leq \frac{P_{h}\left(q^{*}+1\right)-P_{h}\left(q^{*}\right)}{P_{e}\left(q^{*}\right)-P_{e}\left(q^{*}+1\right)} .
$$

The conditions in Proposition 2 are not intuitive, but the implications are reasonable and practical. Let $g(q)=\frac{P_{h}(q)-P_{h}(q-1)}{P_{e}(q-1)-P_{e}(q)}$, then $g(q+1)=\frac{P_{h}(q+1)-P_{h}(q)}{P_{e}(q)-P_{e}(q+1)} \cdot g(q)$ means the ratio of the increase of customer loss probability at front desk to the decrease of customer loss probability at online platform, if the quantity of OERs is increased from $q-1$ to $q$. In (9), $\frac{(1-\theta) w_{e} \lambda_{e}}{w_{h} \lambda_{h}}$, which is equal to $\frac{(1-\theta) w_{e} \lambda_{e} / \mu}{w_{h} \lambda_{h} / \mu}$, means the ratio of the expected revenue per day from the online platform to that from the front desk with no customer loss. Because $P_{e}(q)$ and $P_{h}(q)$ are decreasing and increasing in $q$, respectively [23], and $P_{e}(q)$ and $P_{h}(q)$ are convex functions of $q$ (see the proof of Proposition 1), then $g(q)$ is increasing in $q$. The left inequality in (9), $g\left(q^{*}\right) \leq \frac{(1-\theta) w_{e} \lambda_{e}}{w_{h} \lambda_{h}}$, is equivalent to $\left[P_{h}\left(q^{*}\right)-P_{h}\left(q^{*}-1\right)\right] w_{h} \lambda_{h} \leq\left[P_{e}\left(q^{*}-1\right)-P_{e}\left(q^{*}\right)\right](1-\theta) w_{e} \lambda_{e}$. It implies that the increase of expected revenue loss per day from the front desk is less than the decrease of expected revenue loss from the online platform, if the quantity of OERs is increased from $q^{*}-1$ to $q^{*}$. The right inequality in (9), $\frac{(1-\theta) w_{e} \lambda_{e}}{w_{h} \lambda_{h}} \leq g\left(q^{*}+1\right)$, is equivalent to $\left[P_{h}\left(q^{*}+1\right)-P_{h}\left(q^{*}\right)\right] w_{h} \lambda_{h} \geq\left[P_{e}\left(q^{*}\right)-P_{e}\left(q^{*}+1\right)\right](1-\theta) w_{e} \lambda_{e}$. It implies that the increase of expected revenue loss per day from the front desk is larger than the decrease of expected revenue loss from the online platform, if the quantity of OERs is increased from $q^{*}$ to $q^{*}+1$. Since $g(q)$ is increasing in $q$, the two inequalities in (9) ensure that $q^{*}$ is the optimal quantity of OERs which maximizes the expected revenue of the hotel.

Up till now we are able to answer the second question proposed in Section 3.1. However, to answer the first question, we need to investigate the "complete sharing" case, and do some analysis on the property of the optimal solution of Model (7).

\subsection{Model for "Complete Sharing"}

In the "complete sharing" case, the available room in the hotel will be provided to the customer whose booking request comes first, either from the front desk or from the online platform. To the hotel, these two customer flows can be integrated to one new customer flow. Based on the assumptions that these two customer flows are independent Poisson arrival process, with the arrival rate of $\lambda_{h}$ and $\lambda_{e}$, respectively, the new customer arrival process is also a Poisson process and the arrival rate is $\Lambda\left(=\lambda_{h}+\lambda_{e}\right)$ [24]. Since it is assumed that the time customers stay in the hotel is an i.i.d. random variable and follows a general distribution, the front desk together with the online platform is an $M / G / Q / Q$ queue system.

Let $\tilde{P}$ be the probability of all rooms taken in the $M / G / Q / Q$ system, then

$$
\tilde{P}=\frac{\left(\lambda_{h}+\lambda_{e}\right)^{Q}}{\mu^{Q} Q !} \frac{1}{\sum_{k=0}^{Q} \frac{\left(\lambda_{h}+\lambda_{e}\right)^{k}}{\mu^{k} k !} .}
$$

For the convenience of comparison, we let the booking prices and the commission proportion remain the same for both the "setting OERs" case and the "complete sharing" case. Let $\tilde{\pi}$ be the expected revenue of the hotel in the "complete sharing" case. $\tilde{\pi}$ can be computed as follows.

$$
\tilde{\pi}=\frac{w_{h} \lambda_{h}+(1-\theta) w_{e} \lambda_{e}}{\mu}(1-\tilde{P})=\frac{w_{h} \lambda_{h}+(1-\theta) w_{e} \lambda_{e}}{\mu}\left(1-\frac{\left(\lambda_{h}+\lambda_{e}\right)^{Q}}{\mu^{Q} Q !} \frac{1}{\sum_{k=0}^{Q} \frac{\left(\lambda_{h}+\lambda_{e}\right)^{k}}{\mu^{k} k !}}\right) .
$$


It is obvious from Equation (11) that $\tilde{\pi}$ has no relationship with $q$. That's why we set the "complete sharing” case as the benchmark.

\section{Property Analysis}

To find out when should a hotel set OERs and when a hotel should share all room resources with the online platform, we check the relative magnitude of the expected revenue of the hotel under "setting OERs" and "complete sharing" cases. Define a variable $\alpha$ to be the ratio of these two expected revenues, i.e.,

$$
\alpha=\frac{\pi_{h}\left(q^{*}\right)}{\tilde{\pi}} .
$$

when $\alpha>1$, the hotel is more willing to set OERs. And when $\alpha<1$, the hotel is more willing to share all room resources with the online platform.

Since $\pi_{h}\left(q^{*}\right)$ depends on $q^{*}, \alpha$ also depends on $q^{*}$. Thus in the remaining part of this section, we try to finish two tasks. One is to find out what variables affect the optimal quantity of OERs, $q^{*}$, and what the effects are. The other is to find out what variables affect the revenue ratio, $\alpha$, and what the effects are.

Specifically, we check the effects of the following four factors on $q^{*}$ and $\alpha$ : the booking prices ( $w_{h}$ and $\left.w_{e}\right)$, the proportion of the booking price as the commission $(\theta)$, the customer arriving rates $\left(\lambda_{h}\right.$ and $\left.\lambda_{e}\right)$, and the expected customer stay time in the hotel $(1 / \mu)$.

\subsection{The Factor of Booking Prices ( $w_{h}$ and $w_{e}$ )}

For the effects of booking prices ( $w_{h}$ and $w_{e}$ ) on $q^{*}$, we have the following proposition.

Proposition 3: The optimal quantity of OERs, decreases in $w_{h}$, and increases in $w_{e}$.

Proposition 3 can be explained as follows. When the other parameters are given, if $w_{h}$ is increased, the potential revenue from a room booked at the hotel's front desk is increased. Thus the hotel is more willing to limit rooms for booking at the online platform, i.e. setting less OERs. However, if $w_{e}$ is increased, the revenue from a room booked at the online platform front desk is increased. Thus the hotel is willing to offer more OERs to the online platform.

Proposition 3 addresses how $q^{*}$ will be changed when one booking price $\left(w_{h}\right.$ or $\left.w_{e}\right)$ is changed given the other fixed. However, when the two prices are changed simultaneously, it is difficult to find conclusions analytically. Thus we use numerical studies to analyze it. Let $\lambda_{h}=30, \lambda_{e}=20, Q=100, \mu=0.5$ and $\theta=0.2$. The effects of $w_{h}$ and $w_{e}$ on $q^{*}$ are illustrated in Figure 2.

Figure 2 shows that the conclusions in Proposition 3 are valid. Furthermore, it can be observed from Figure 2 that, if the ratio of $w_{h}$ to $w_{e}$ is increased, $q^{*}$ tends to be lower. If the ratio of $w_{h}$ to $w_{e}$ is decreased, $q^{*}$ tends to be higher. These two observations are reasonable, because higher ratio of $w_{h}$ to $w_{e}$ means less revenue from the online platform. Thus the hotel tends to decrease OERs.

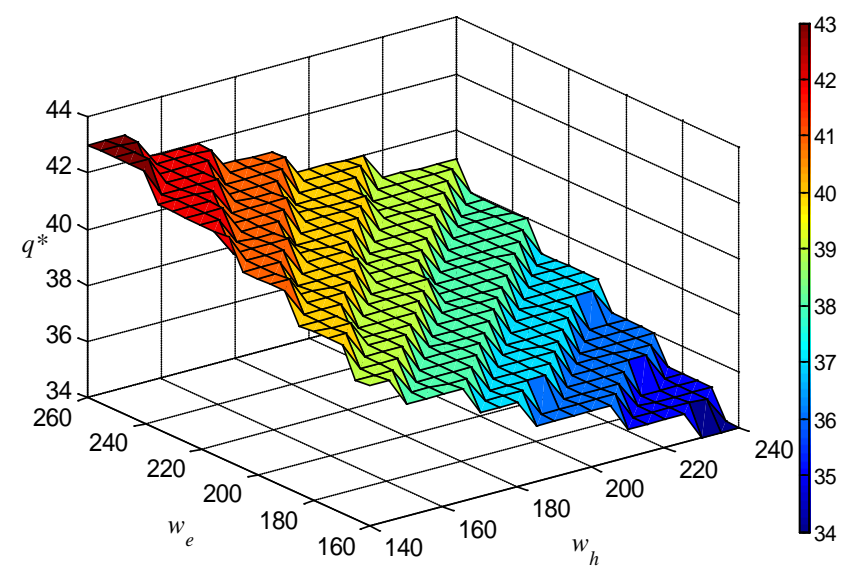

Figure 2. The effect of $w_{h}$ and $w_{e}$ on $q^{*}$. 
It is rather difficult to analytically identify the effects of $w_{h}$ and $w_{e}$ on $\alpha$, since $\pi_{h}\left(q^{*}\right)$ is difficult to be analytically solved. We use numerical studies to check the effects. Let $\lambda_{h}=30, \lambda_{e}=20, Q=100$, $\mu=0.5$ and $\theta=0.2$. The effects of $w_{h}$ and $w_{e}$ on $\alpha$ are illustrated in Figure 3 .

It is shown in Figure 3 that $\alpha$ increases in $w_{h}$, and decreases in $w_{e}$. Furthermore, $\alpha$ increases in the ratio of $w_{h}$ to $w_{e}$. These observations are reasonable, because on one hand, as $w_{e}$ increases, the hotel can get more revenue from booking out one room at the online platform. Thus the hotel is more willing to share more rooms with the online platform, and then the "complete sharing" case is welcome. As $w_{e}$ decreases, the hotel is more willing to set OERs. It is important to note that the expected revenue of the hotel in "setting OERs" case can be higher than that in "complete sharing" case, when the ratio of $w_{h}$ to $w_{e}$ is rather large. This indicates that in particular case, "complete sharing" form may not be better than "setting OERs" form for the hotel.

\subsection{The Factor of the Proportion of Booking Price as Commission $(\theta)$}

For the effect of the proportion of booking price as commission $(\theta)$ on $q^{*}$, we have the following proposition.

Proposition 4. The optimal quantity of OERs, $q^{*}$, decreases in $\theta$.

The conclusion in Proposition 4 can be interpreted as follows. Larger $\theta$ means the hotel should give more commission to the online platform, which implies less revenue from booking out one room at the online platform. Thus the hotel tends to set less OERs when $\theta$ is increased.

To show the effect of $\theta$ on $q^{*}$ numerically, we also do some computation. Let $\lambda_{h}=30, \lambda_{e}=20$, $w_{h}=200, w_{e}=180, Q=100$, and $\mu=0.5$. The effect of the proportion of the booking price as the commission, $\theta$, on $q^{*}$ is illustrated in Figure 4 .

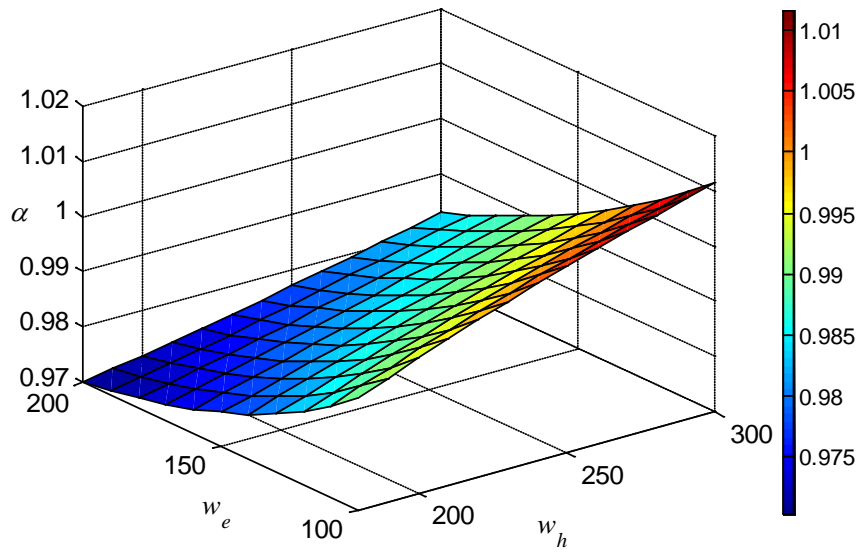

Figure 3. The effect of $w_{h}$ and $w_{e}$ on $\alpha$.

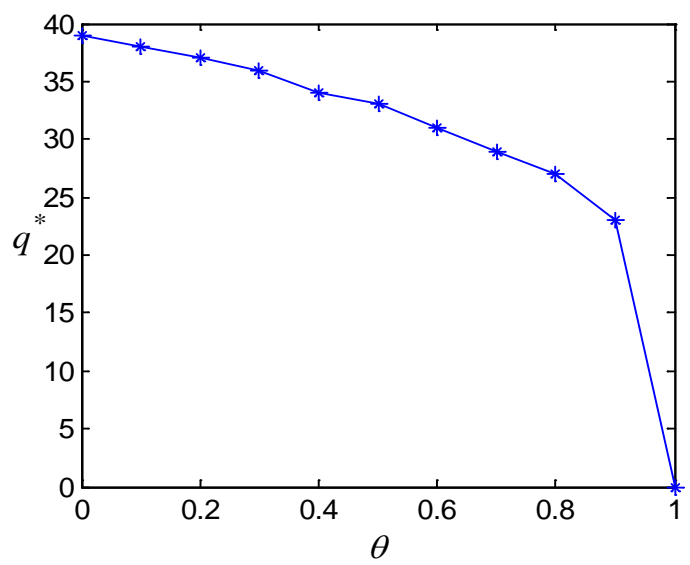

Figure 4. The effect of $\theta$ on $q^{*}$. 
Figure 4 shows that $q^{*}$ decreases in $\theta$, which verifies the conclusion in Proposition 4 . Let $\lambda_{h}=30$, $\lambda_{e}=20, w_{h}=200, w_{e}=180, Q=100$, and $\mu=0.5$. We check the effect of $\theta$ on $\alpha$, as shown in Figure 5 .

Figure 5 shows that $\alpha$ increases in $\theta$, which means as $\theta$ increases, the hotel is less willing to share all room resources with the online platform but like to set OERs. This is reasonable because higher $\theta$ means less revenue from booking out one room at the online platform.

\subsection{The Factor of Customer Arriving Rate $\left(\lambda_{h}\right.$ and $\left.\lambda_{e}\right)$}

It is difficult to analytically check the effect of $\lambda_{h}$ and $\lambda_{e}$ on $q^{*}$ and $\alpha$. We use numerical studies to check these effects. Let $w_{h}=200, w_{e}=180, Q=100, \mu=0.5$ and $\theta=0.2$. The effects of $\lambda_{h}$ and $\lambda_{e}$ on $q^{*}$ are illustrated in Figure 6.

Figure 6 shows that $q^{*}$ decreases in $\lambda_{h}$ and increases in $\lambda_{e}$. Furthermore, $q^{*}$ decreases in the ratio of $\lambda_{h}$ to $\lambda_{e}$. This is because larger $\lambda_{e}$ means more customers arriving at online platform, then it will be better to share more rooms with the online platform. However, larger $\lambda_{h}$ means more customers arriving at the front desk. Since the total rooms are fixed, it will be better to set smaller OERs.

For the effect of $\lambda_{h}$ and $\lambda_{e}$ on $\alpha$, it is not easy to make a speculation. We also resort to numerical studies. Let $w_{h}=200, w_{e}=180, Q=100, \mu=0.5$ and $\theta=0.2$. The effects of $\lambda_{h}$ and $\lambda_{e}$ on $\alpha$ are illustrated in Figure 7.

As shown in Figure 7, the effects of $\lambda_{h}$ and $\lambda_{e}$ on $\alpha$ are a little complicated. When both $\lambda_{h}$ and $\lambda_{e}$ are low, $\alpha$ is close to 1 . It means the expected revenue of the hotel is nearly the same in both the "setting OERs" case and the "complete sharing" case, because there is little probability that all rooms are taken. When

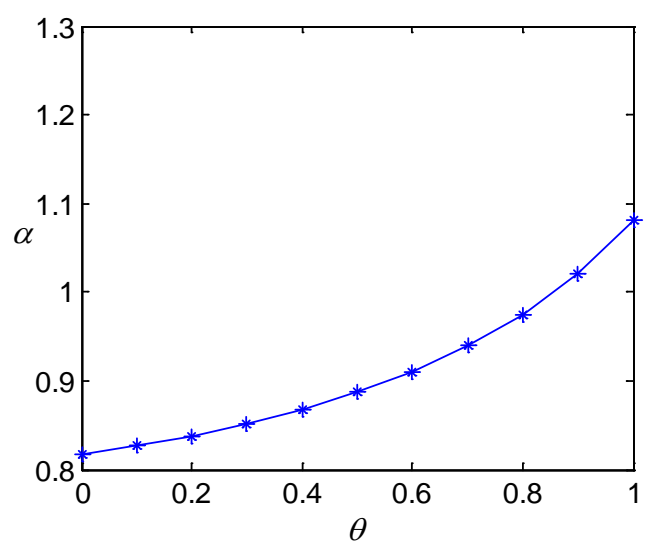

Figure 5. The effect of $\theta$ on $\alpha$.

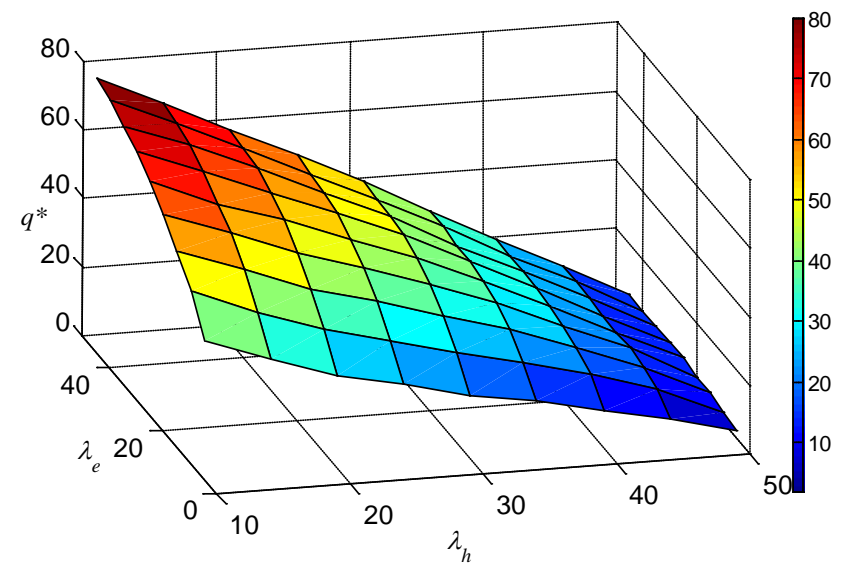

Figure 6. The effects of $\lambda_{h}$ and $\lambda_{e}$ on $q^{*}$. 
one of $\lambda_{h}$ and $\lambda_{e}$ is high and the other is low, $\alpha$ is less than 1 . This is because in the "complete sharing" case, all room resources can be fully utilized. But the resource utilization may not be so high in the "setting OERs" case. Thus the "complete sharing" case is better than the "setting OERs" case. If both $\lambda_{h}$ and $\lambda_{e}$ is high, $\alpha$ is higher than 1 . This is because in this situation, the hotel can get highest revenue by setting a small quantity of OERs. However, if the hotel shares all room resources with online platform, many rooms may be occupied by online customer and the hotel will have potential revenue loss.

\subsection{The Factor of Expected Customer Stay Time in the Hotel $(1 / \mu)$}

We resort to numerical studies to check the effects of $1 / \mu$ on $q^{*}$ and $\alpha$. Let $\lambda_{h}=40, \lambda_{e}=30, w_{h}=200$, $w_{e}=180, Q=100$ and $\theta=0.2$. The effect of the expected customer stay time $(1 / \mu)$ on $q^{*}$ is illustrated in Figure 8.

It can be observed from Figure 8 that $q^{*}$ decreases in the expected customer stay time, $1 / \mu$. This is because, as the expected customer stay time increases, the probability of all room taken in front desk is increasing. It is better to set more HERs and less OERs. When the expected customer stay time is rather long ( 3 or more days in this example), the optimal quantity of OERs is 0 , since the probability of all room taken in front desk is close to 1 , and there is no need to provide OERs to online platform. This is actually the third case, i.e. the "operating alone" case. In this case, the hotel operates alone, without cooperating with the online platform. This case is a special one of the "setting OERs" case.

The corresponding effect of $1 / \mu$ on $\alpha$ is illustrated in Figure 9.

It can be observed from Figure 9 that $\alpha$ is increasing in the expected customer stay time, $1 / \mu$. This can be explained similarly. As the expected customer stay time increases, the probability of all room taken in front desk

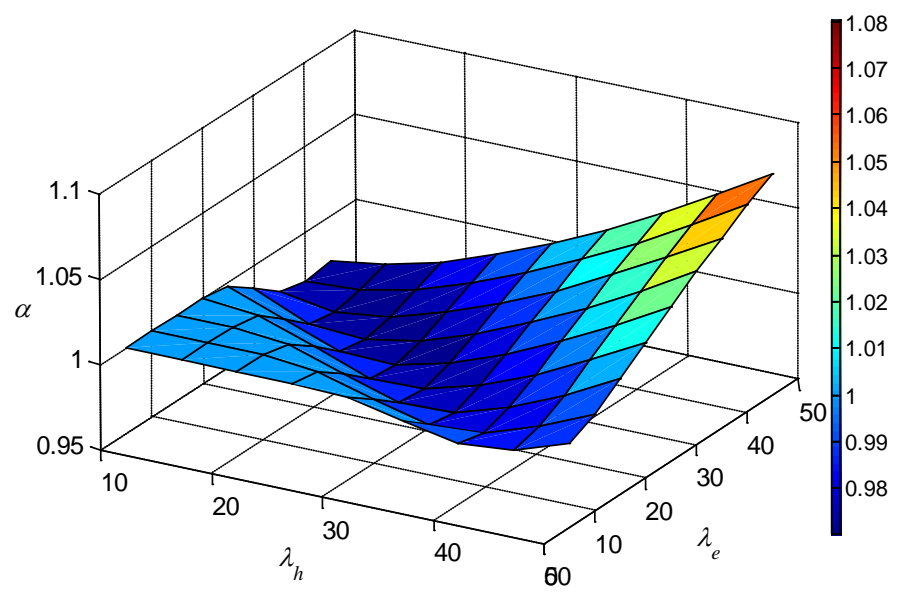

Figure 7. The effects of $\lambda_{h}$ and $\lambda_{e}$ on $\alpha$.

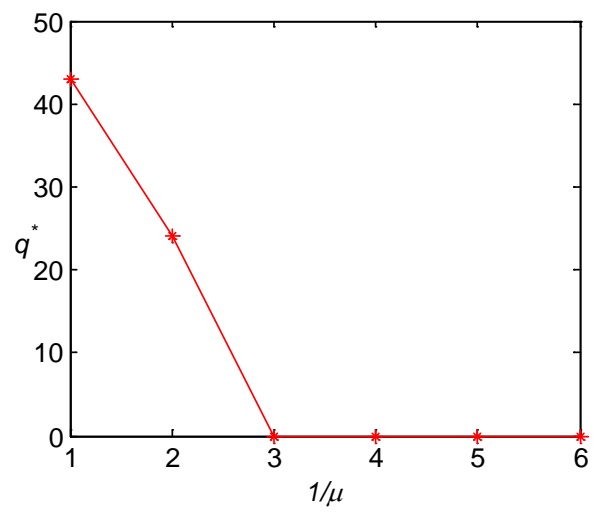

Figure 8. The effect of $1 / \mu$ on $q^{*}$. 


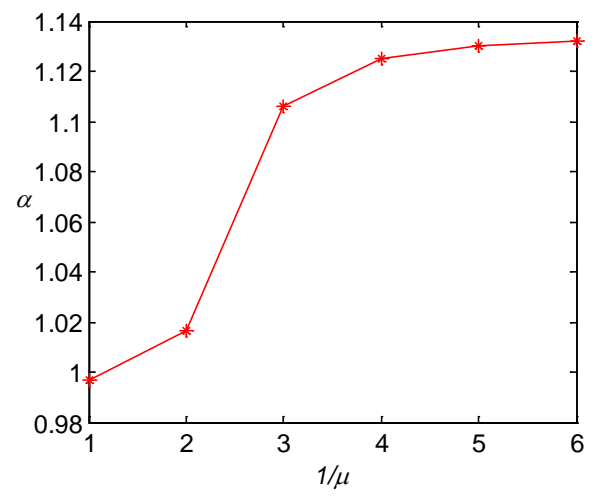

Figure 9. The effect of $1 / \mu$ on $\alpha$.

is increasing. It is better to share less room with the online platform, i.e. "complete sharing" is not welcome to the hotel.

\section{Conclusions}

In this paper, we have investigated a new type of hotel room management problem in the coming E-commerce age: deciding whether to set a fixed quantity of Online-Exclusive-Rooms (OERs) for booking at the online platform, and if yes, then how many OERs should be set? This "setting OER" form is compared with another intuitive cooperating form, complete sharing, i.e. the hotel shares all room resources with the online platform for booking. We formulate the hotel and the online platform as a loss queueing service system. Properties of the expected revenue of the hotel and the optimal quantity of OERs to maximize the hotel's revenue are identified.

In the "setting OER" form, the expected revenue of the hotel is proved to be a concave function of the quantity of OERs, which insures that there is an optimal quantity of OERs which maximizes the expected revenue of the hotel. The method of determining the optimal quantity of OERs is presented. It is found by analytical and numerical studies that: 1) the optimal quantity of OERs decreases in the booking price at the front desk of the hotel, and increases in the booking price at the online platform; 2) the optimal quantity of OERs decreases in the proportion of booking price as commission; 3) the optimal quantity of OERs decreases in the customer arrival rate at the front desk, and increases in the customer arrival rate at the online platform; 4) the optimal quantity of OERs decreases in the expected customer stay time. These properties can provide suggestions on how to set and change the optimal quantity of OERs to maximize the expected revenue of the hotel.

It seems that the "complete sharing" form can utilize the capacity of the hotel more fully. The numerical studies in this paper verified this point. However, it is also shown by numerical studies that completely sharing rooms with online platform is not always a good idea for the hotel. It is better to set OERs when the online booking price is too low, or the commission part is too high, or both online and offline customer arrival rates are large, or the expected customer stay time is long.

There are several limitations in this paper. First, the booking price of the OERs is exogenously fixed but not a decision variable; we do not consider the pricing behavior of the online platform. Second, since this paper focuses on the profit optimization of the hotel, we do not consider the behaviors of the online platform, such as advertizing and ranking which can attract customers to book from the online platform. Third, we assume that no more customers can book rooms from the online platform once all OERs are booked out. Actually, some customers still can get rooms in the above case, but with a lower probability. These limitations are all interesting future research directions. Furthermore, if the hotel adopts the "complete sharing" form, how should the expected revenue of the hotel change in the long run? This should also be an interesting topic in the future.

\section{References}

[1] Dellarocas, C. (2003) The Digitization of Word-of-Mouth: Promise and Challenges of Online Feedback Mechanisms. Management Science, 49, 1407-1424. http://dx.doi.org/10.1287/mnsc.49.10.1407.17308

[2] China Tourism Academy (2011) Report of the Development of China’s Tourism E-Commerce. http://www.ctaweb.org/html/2011-9/2011-9-14-17-14-34961.html 
[3] Morgan, N.P. and Piggott, A.R. (2003) Destination Branding and the Role of the Stakeholders: The Case of New Zealand. Journal of Vacation Marketing, 9, 285-299. http://dx.doi.org/10.1177/135676670300900307

[4] O’Connor, P. and Frew, J.A. (2004) An Evaluation Methodology for Hotel Electronic Channels of Distribution. International Journal of Hospitality Management, 23, 179-199. http://dx.doi.org/10.1016/j.ijhm.2003.10.002

[5] Rothstein, M. (1974) Hotel Overbooking as a Markovian Sequential Decision Process. Decision Science, 5, $289-404$. http://dx.doi.org/10.1111/j.1540-5915.1974.tb00624.x

[6] Liberman, V. and Yechialli, U. (1978) On the Hotel Overbooking Problem: An Inventory System with Stochastic Cancellations. Management Science, 24, 1117-1126. http://dx.doi.org/10.1287/mnsc.24.11.1117

[7] Sieburgh, J.A. (1988) Yield Management at Work at Royal Sonesta. Lodging Hospitality, 9, 235-237.

[8] Badinelli, R.D. (2000) An Optimal, Dynamic Policy for Hotel Yield Management. European Journal of Operational Research, 121, 476-503. http://dx.doi.org/10.1016/S0377-2217(99)00046-6

[9] Bitran, G. and Mondschein, S.V. (1995) An Application of Yield Management to the Hotel Industry Considering Multiple Stays. Operation Research, 43, 427-443. http://dx.doi.org/10.1287/opre.43.3.427

[10] Bitran, G. and Gilbert, S.M. (1996) Managing Hotel Reservations with Uncertain Arrivals. Operation Research, 44, 35-49. http://dx.doi.org/10.1287/opre.44.1.35

[11] Baker, R.D. and Collier, D.A. (2003) The Benefits of Optimizing Prices to Manage Demand in Hotel Revenue Management Systems. Production and Operations Management, 12, 502-518. http://dx.doi.org/10.1111/j.1937-5956.2003.tb00217.x

[12] Chiang, W.K., Chhajed, D. and Hess, J. (2003) Direct Marketing, Indirect Profits: A Strategic Analysis of Dual-Channel Supply-Chain Design. Management Science, 49, 1-20. http://dx.doi.org/10.1287/mnsc.49.1.1.12749

[13] Tsay, A. and Agrawal, N. (2004) Channel Conflict and Coordination in the E-Commerce Age. Production and Operations Management, 13, 93-110. http://dx.doi.org/10.1111/j.1937-5956.2004.tb00147.x

[14] Viswanathan, S. (2005) Competing across Technology-Differentiated Channels: The Impact of Network Externalities and Switching Costs. Management Science, 51, 483-496. http://dx.doi.org/10.1287/mnsc.1040.0338

[15] Dumrongsiri, A., Fan, M., Jain, A. and Moinzadeh, K. (2008) A Supply Chain Model with Direct and Retail Channels. European Journal of Operational Research, 187, 691-718. http://dx.doi.org/10.1016/j.ejor.2006.05.044

[16] Miller, B.L. (1969) A Queuing Reward System with Several Customer Classes. Management Science, 16, $234-245$. http://dx.doi.org/10.1287/mnsc.16.3.234

[17] Printezis, A., Burnetas, A. and Mohan, G. (2009) Pricing and Capacity Allocation under Asymmetric Information Using Paris Metro Pricing. International Journal of Operational Research, 5, 265-279. http://dx.doi.org/10.1504/IJOR.2009.025196

[18] Kimura, T. (2000) Equivalence Relations in the Approximations for the $M / G / s / s+r$ Queue. Mathematical and Computer Modeling, 31, 215-224. http://dx.doi.org/10.1016/S0895-7177(00)00090-X

[19] Medhi, J. (2003) Stochastic Models in Queueing Theory. Academic Press, Boston.

[20] Hassin, R. and Haviv, M. (2003) To Queue or Not to Queue: Equilibrium Behavior in Queuing Systems. Kluwer Academic Publishers, Boston. http://dx.doi.org/10.1007/978-1-4615-0359-0

[21] Kleinrock, L. (1975) Queueing Systems. Vol. I, Wiley, New York.

[22] Fox, B. (1966) Discrete Optimization via Marginal Analysis. Management Science, 13, 210-216. http://dx.doi.org/10.1287/mnsc.13.3.210

[23] Harel, A. (1990) Convexity Properties of the Erlang Loss Formula. Operations Research, 38, 499-505. http://dx.doi.org/10.1287/opre.38.3.499

[24] Rabi, N.B. and Edward, C.W. (2009) Stochastic Processes with Applications. Society for Industrial and Applied Mathematics, Philadelphia.

[25] Messerli, E.J. (1972) Proof of a Convexity Property of the Erlang B Formula. The Bell System Technical Journal, 51, 951-953. http://dx.doi.org/10.1002/j.1538-7305.1972.tb01956.x

[26] Boyd, S. and Vandenberghe, L. (2004) Convex Optimization. Cambridge University Press, Cambridge. http://dx.doi.org/10.1017/CBO9780511804441 


\section{Appendix}

\section{Proof of Proposition 1}

In an $M / G / c / c$ queueing system, the probability of new customer rejected because all servers are busy, is a convex function of the number of servers [25]. That means in our problem, $P_{e}(q)$ is a convex function of $q$.

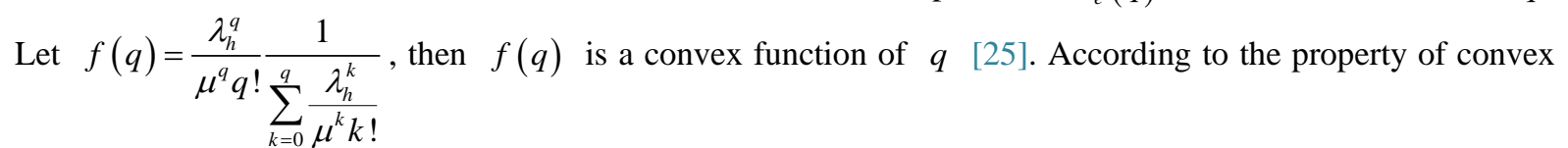

function [26], $f(Q-q)$ is also a convex function of $q$. Thus $P_{h}(q)$, which is equal to $f(Q-q)$, is a convex function of $q$. Because the additive inverse of a convex function is a concave function [26], $-P_{h}(q)$ and $-P_{e}(q)$ are concave functions of $q$. In Equation (5), $w_{h}, w_{e}, \lambda_{h}, \lambda_{e}, \theta$ and $\mu$ are fixed positive constants. Thus as a linear combination of $-P_{h}(q)$ and $-P_{e}(q), \pi_{h}(q)$ is a concave function of the quantity of OERs, $q$.

\section{Proof of Proposition 2}

From (5), we have $\pi_{h}(q)=\frac{w_{h} \lambda_{h}}{\mu}\left[1-P_{h}(q)\right]+\frac{(1-\theta) w_{e} \lambda_{e}}{\mu}\left[1-P_{e}(q)\right]$. Submitting this Equation into (8), we have

$$
\left\{\begin{array}{l}
\frac{w_{h} \lambda_{h}}{\mu}\left(1-P_{h}\left(q^{*}\right)\right)+\frac{(1-\theta) w_{e} \lambda_{e}}{\mu}\left(1-P_{e}\left(q^{*}\right)\right) \geq \frac{w_{h} \lambda_{h}}{\mu}\left(1-P_{h}\left(q^{*}-1\right)\right)+\frac{(1-\theta) w_{e} \lambda_{e}}{\mu}\left(1-P_{e}\left(q^{*}-1\right)\right) \\
\frac{w_{h} \lambda_{h}}{\mu}\left(1-P_{h}\left(q^{*}\right)\right)+\frac{(1-\theta) w_{e} \lambda_{e}}{\mu}\left(1-P_{e}\left(q^{*}\right)\right) \geq \frac{w_{h} \lambda_{h}}{\mu}\left(1-P_{h}\left(q^{*}+1\right)\right)+\frac{(1-\theta) w_{e} \lambda_{e}}{\mu}\left(1-P_{e}\left(q^{*}+1\right)\right) .
\end{array}\right.
$$

Simplify the above Equation, and we get

$$
\left\{\begin{array}{l}
\frac{w_{h} \lambda_{h}}{\mu} P_{h}\left(q^{*}\right)+\frac{(1-\theta) w_{e} \lambda_{e}}{\mu} P_{e}\left(q^{*}\right) \leq \frac{w_{h} \lambda_{h}}{\mu} P_{h}\left(q^{*}-1\right)+\frac{(1-\theta) w_{e} \lambda_{e}}{\mu} P_{e}\left(q^{*}-1\right) \\
\frac{w_{h} \lambda_{h}}{\mu} P_{h}\left(q^{*}\right)+\frac{(1-\theta) w_{e} \lambda_{e}}{\mu} P_{e}\left(q^{*}\right) \leq \frac{w_{h} \lambda_{h}}{\mu} P_{h}\left(q^{*}+1\right)+\frac{(1-\theta) w_{e} \lambda_{e}}{\mu} P_{e}\left(q^{*}+1\right) .
\end{array}\right.
$$

After further simplification, we have

$$
\left\{\begin{array}{l}
\frac{P_{h}\left(q^{*}\right)-P_{h}\left(q^{*}-1\right)}{P_{e}\left(q^{*}-1\right)-P_{e}\left(q^{*}\right)} \leq \frac{(1-\theta) w_{e} \lambda_{e}}{w_{h} \lambda_{h}} \\
\frac{P_{h}\left(q^{*}+1\right)-P_{h}\left(q^{*}\right)}{P_{e}\left(q^{*}\right)-P_{e}\left(q^{*}+1\right)} \geq \frac{(1-\theta) w_{e} \lambda_{e}}{w_{h} \lambda_{h}} .
\end{array}\right.
$$

which implies that $\frac{P_{h}\left(q^{*}\right)-P_{h}\left(q^{*}-1\right)}{P_{e}\left(q^{*}-1\right)-P_{e}\left(q^{*}\right)} \leq \frac{(1-\theta) w_{e} \lambda_{e}}{w_{h} \lambda_{h}} \leq \frac{P_{h}\left(q^{*}+1\right)-P_{h}\left(q^{*}\right)}{P_{e}\left(q^{*}\right)-P_{e}\left(q^{*}+1\right)}$.

\section{Proof of Proposition 3}

Denote that $\Delta \pi(q)=\pi_{h}(q)-\pi_{h}(q-1), \Delta P_{h}(q)=P_{h}(q)-P_{h}(q-1)$, and $\Delta P_{e}(q)=P_{e}(q)-P_{e}(q-1)$. Since $P_{h}(q)$ strictly increases in $q$ and $P_{e}(q)$ strictly decreases in $q$ [25], we have $\Delta P_{h}(q)>0$ and $\Delta P_{e}(q)<0$.

According to Equation (5), we have

$$
\Delta \pi(q)=-\Delta P_{h}(q) \frac{w_{h} \lambda_{h}}{\mu}-\Delta P_{e}(q) \frac{(1-\theta) w_{e} \lambda_{e}}{\mu} .
$$


Consider two prices of an HER, $w_{h 1}$ and $w_{h 2}\left(w_{h 1}>w_{h 2}\right)$. Let $q_{1}^{*}$ and $q_{2}^{*}$ be the optimal quantity of OERs when the prices of an HER are $w_{h 1}$ and $w_{h 2}$, respectively.

It is easy to verify that $\left.\Delta \pi(q)\right|_{w_{h}=w_{h 1}}-\left.\Delta \pi(q)\right|_{w_{h}=w_{h 2}}=\Delta P_{h}(q) \frac{\lambda_{h}\left(w_{h 2}-w_{h 1}\right)}{\mu}<0$, which implies

$$
\left.\Delta \pi\left(q_{1}^{*}\right)\right|_{w_{h}=w_{h 1}}-\left.\Delta \pi\left(q_{1}^{*}\right)\right|_{w_{h}=w_{h 2}}<0,
$$

and

$$
\left.\Delta \pi\left(q_{2}^{*}\right)\right|_{w_{h}=w_{h 1}}-\left.\Delta \pi\left(q_{2}^{*}\right)\right|_{w_{h}=w_{h 2}}<0 .
$$

According to Proposition $1, \pi_{h}(q)$ is a concave function of $q$, thus $\Delta \pi(q)$ decreases in $q$. Since $\pi_{h}(q)$ achieves maximum at $q^{*}$, then $q^{*}=\arg \min _{0 \leq q \leq Q}\{\Delta \pi(q) \mid \Delta \pi(q)>0\}$. Because $q_{1}^{*}$ and $q_{2}^{*}$ are the optimal quantities of OERs when the prices of an HER are $w_{h 1}$ and $w_{h 2}$, respectively, we have $\left.\Delta \pi\left(q_{1}^{*}\right)\right|_{w_{h}=w_{h 1}}>0$. Furthermore, according to (A.2), $\left.\Delta \pi\left(q_{1}^{*}\right)\right|_{w_{h}=w_{h 2}}>0$.

Since $q_{2}^{*}=\arg \min _{0 \leq q \leq Q}\left\{\Delta \pi(q) \mid \Delta \pi(q)>0 ; w_{h}=w_{h 2}\right\}$, we have $\left.\Delta \pi\left(q_{2}^{*}\right)\right|_{w_{h}=w_{h 2}} \leq\left.\Delta \pi\left(q_{1}^{*}\right)\right|_{w_{h}=w_{h 2}}$. Because $\Delta \pi(q)$ decreases in $q$, we have $q_{2}^{*} \geq q_{1}^{*}$, which implies $q^{*}$ decreases in $w_{h}$.

Following the similar way, $q^{*}$ increases in $w_{e}$. Thus we omit it.

\section{Proof of Proposition 4}

According to the proof of Proposition 3 (Equation A.1), we have

$$
\Delta \pi(q)=-\Delta P_{h}(q) \frac{w_{h} \lambda_{h}}{\mu}-\Delta P_{e}(q) \frac{(1-\theta) w_{e} \lambda_{e}}{\mu} .
$$

Consider two proportions of the booking price as the commission, $\theta_{1}$ and $\theta_{2}\left(\left(\theta_{1}>\theta_{2}\right)\right)$. Let $q_{1}^{*}$ and $q_{2}^{*}$ be the optimal quantity of OERs when the proportions are $\theta_{1}$ and $\theta_{2}$, respectively.

Since $\Delta P_{e}(q)<0$, It is easy to verify that $\left.\Delta \pi(q)\right|_{\theta=\theta_{1}}-\left.\Delta \pi(q)\right|_{\theta=\theta_{2}}=\Delta P_{e}(q) \frac{w_{e} \lambda_{e}\left(\theta_{1}-\theta_{2}\right)}{\mu}<0$, which implies

$$
\left.\Delta \pi\left(q_{1}^{*}\right)\right|_{\theta=\theta_{1}}-\left.\Delta \pi\left(q_{1}^{*}\right)\right|_{\theta=\theta_{2}}<0
$$

Because $q^{*}=\arg \min _{0 \leq q \leq Q}\{\Delta \pi(q) \mid \Delta \pi(q)>0\}$, we have $\left.\Delta \pi\left(q_{1}^{*}\right)\right|_{\theta=\theta_{1}}>0$. Furthermore, according to (A.5), $\left.\Delta \pi\left(q_{1}^{*}\right)\right|_{\theta=\theta_{2}}>0$.

Since $q_{2}^{*}=\arg \min _{0 \leq q \leq Q}\left\{\Delta \pi(q) \mid \Delta \pi(q)>0 ; \theta=\theta_{2}\right\}$, we have $\left.\Delta \pi\left(q_{1}^{*}\right)\right|_{\theta=\theta_{2}} \leq\left.\Delta \pi\left(q_{1}^{*}\right)\right|_{\theta=\theta_{2}}$.

Because $\Delta \pi(q)$ decreases in $q$, we have $q_{2}^{*} \geq q_{1}^{*}$, which implies $q^{*}$ decreases in $\theta$. 
Scientific Research Publishing (SCIRP) is one of the largest Open Access journal publishers. It is currently publishing more than 200 open access, online, peer-reviewed journals covering a wide range of academic disciplines. SCIRP serves the worldwide academic communities and contributes to the progress and application of science with its publication.

Other selected journals from SCIRP are listed as below. Submit your manuscript to us via either submit@scirp.org or Online Submission Portal.
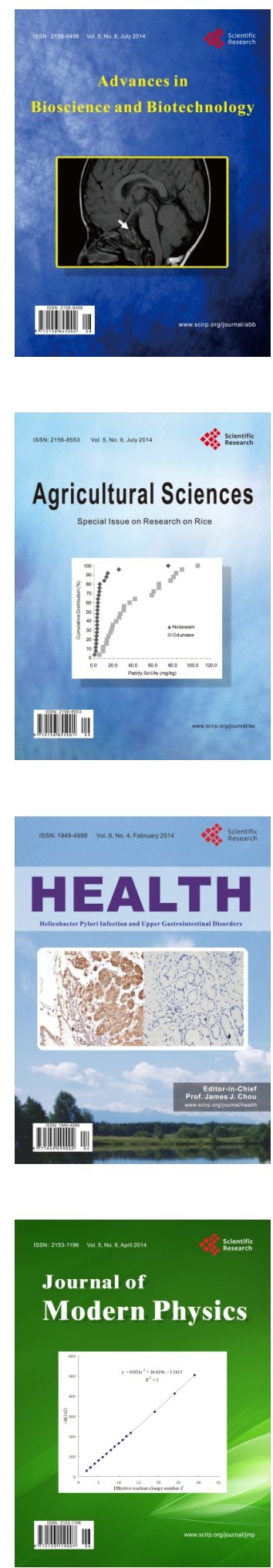
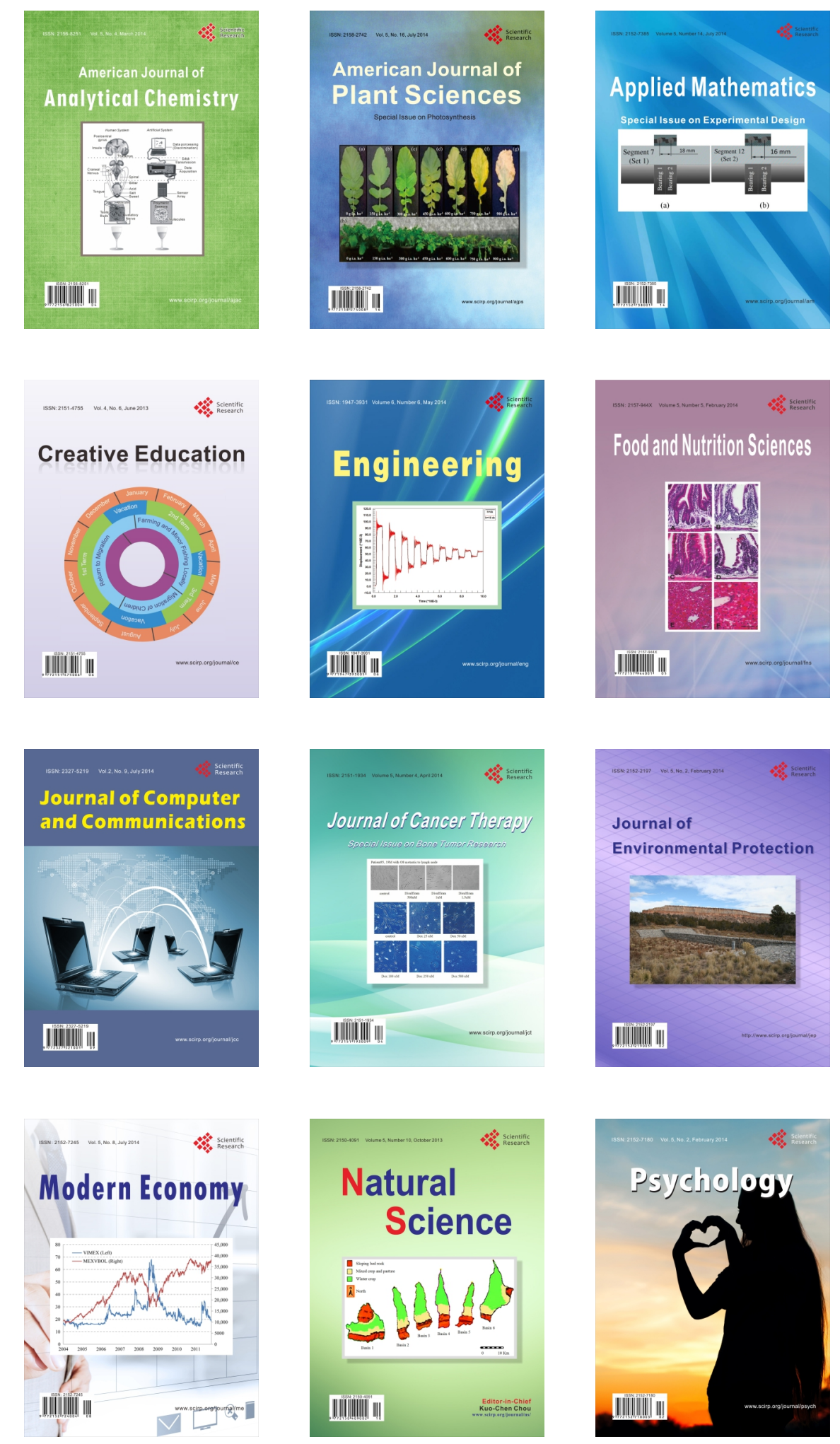